\title{
A mechanical model for cell motility and tissue morphogenesis
}

\author{
S. Jauvert ${ }^{\mathrm{a}, \mathrm{b} *}$, R. Peyroux ${ }^{\mathrm{a}, \mathrm{b}}$ and V. Richefeu ${ }^{\mathrm{a}, \mathrm{b}}$ \\ ${ }^{a}$ Université de Grenoble Alpes, 3SR, F-38041 Grenoble, France; ${ }^{b}$ CNRS, 3SR, F-38041 Grenoble, France
}

Keywords: cell motility; cell adhesion; cell division; tissue morphogenesis; mechanical effects

\section{Introduction}

The mechanical environment strongly influences many cellular behaviours and responses such as spreading, adhesion, migration, proliferation, differentiation and apoptosis (Lauffenburger and Horwitz 1996; Engler et al. 2006; Dieterich et al. 2008; Trepat et al. 2009; Rørth 2011;). This has been extensively reported for a single cell (motility, random walk and division), for the interaction between two cells (cell-to-cell contact and chemotaxis) or at the tissue level (morphogenesis or tumor progression). For example, many experiments produce valuable observations on single-cell migration on a substrate or on collective migration and these works widely use traction force methods. The values of forces exerted by the cells on the substrate were then estimated by coupling microscopy and inversion procedures (Sabass et al. 2008; Ambrosi et al. 2009). Interesting results have also been found regarding the influence of the substrate rigidity and its anisotropy on cell motility (Lo et al. 2000; Saez et al. 2007; Trichet et al. 2012). Other works focused on cell division and enlightened the association between the division orientation and mechanical efforts (Reffay et al. 2011). All these works are concerned with the study of chemical and physical phenomena arising at different scales (filopodia, cytoskeleton and tissue), and consequently with mechanotransduction, referring to the many mechanisms by which cells convert mechanical stimulus into chemical activity.

To better understand the relationships between mechanical and biological effects, some authors have developed models at the cell scale and performed numerical simulations to predict collective effects (Drasdo and Höhme 2005; Peyroux and Pattana 2007; Basan et al. 2011; Vermolen and Gefen 2012). Most of these models can be marked as discrete mechanical models, as distinct from classical continuum mechanics.

\section{Methods}

The present mechanical model of cell motility is based on the dynamics of solid bodies that obeys Newton's laws. A cell placed on a substrate, pictured as a sphere onto a plane, was accelerated under the action of external forces. The latter were either classical frictional contact forces or pulling forces applied by filopodia (or lamellipodia) that bind cells to focal adhesion (FA) areas fixed on the substrate. Assuming a uniform distribution of FA areas in the vicinity of the cell, a pulling force on a FA area represents a mean value of all the actions exerted by the cell on the substrate at this point. Therefore, a cell, surrounded by FA areas, has the ability to remain motionless because of the force balance. To migrate, a cell needs to be polarised. The polarity of a cell can be defined by the direction of the vector that connects the centre of the cell to the position of a chemoattractant, or by the direction of the instantaneous velocity of the cell. According to this polarity definition, a cell can exhibit a targeted trajectory or a random walk. Practically, the polarity of the cell was introduced by imposing an asymmetry in the magnitudes of the pulling forces between the front side and the rear side of the cell.

In addition to the motility, the model can take into account the cell growth and the cell division. Both phenomena were introduced by kinetics laws, consistent with the mitotic cycle for the case of division. The rate of growth and division can be dependent on environmental factors, such as nutrient availability, and can also be connected to mechanical aspects. This last coupling is a key point to obtain mechanical effects on tissue growth and tissue organisation, the forces acting on cells being considered either as an inhibiting or an activating factor for growth and division.

\section{Results and discussion}

One asset of numerical simulation is to provide, at each time, a full description of the positions, velocities and forces existing within the system. The main goal of our work is to connect the mechanical analysis to biological aspects in order to address problems such as morphogenesis of tissue, cell motility, wound healing or tumour development. Two examples are mainly examined.

\footnotetext{
*Corresponding author. Email: sylvie.jauvert@3sr-grenoble.fr 


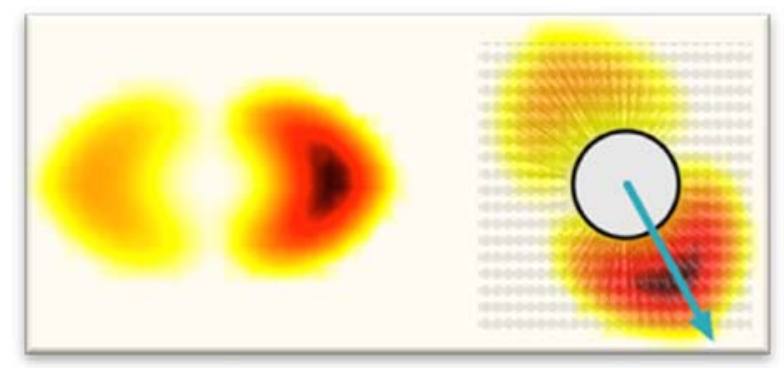

Figure 1. Two cells moving on a functionalised substrate. Colours symbolise force levels. The cell shape, its velocity and the loci of the FA areas are superimposed onto the right cell.

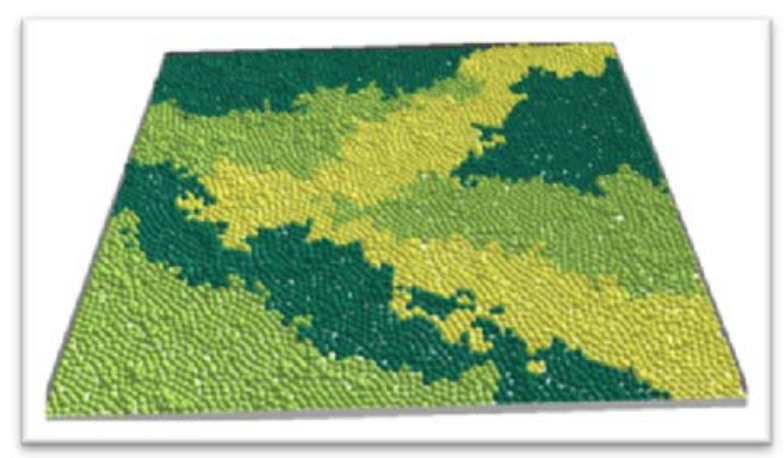

Figure 2. Tissue generation starting from eight stem cells of three different species in a squared box.

The first example is concerned with cell motility. Two cells are placed on a functionalised plane substrate, on which adhesion points are distributed. Depending on initial cell velocity, FA areas position and the ability of the cell to detect FA areas or not, the movement of cells is analysed to characterise random walk features. The diffusive nature of the cell trajectory can thus be discussed, and the map of the forces exerted by the cell on the substrate (Figure 1) can be compared with the results of various experiments.

The second example (Figure 2) shows a tissue generation. The simulation starts with eight cells of three different species in a squared box. They can grow and divide up to fill the entire box (i.e. reaching a given density value). The kinetics of growth and division are connected to the level of efforts between cells. The aim of this simulation was to correlate the final repartition of spheres and species to the kinetics of growth and division and to evaluate the influence of mechanical efforts on the texture of the tissue.

\section{Conclusions}

Our work has been to consider a cell-based model in order to address mechanical problems linked to cell motility and tissue morphogenesis. The global approach consists of modelling cells by spheres, ignoring the complex description of the cytoskeleton and focusing on contact interactions between cell and substrate or between cell and cell, and active forces developed by cells to migrate. Growth and division were also taken into account to approach the morphogenesis of tissue. This model still needs to be enhanced by taking into account the relevant biological items, but here and now, it allows to evaluate mechanical efforts of cells and investigate their collective behaviour.

\section{Acknowledgements}

3SR-Lab is part of Labex TEC 21 (ANR-11-LABX-0030-01).

\section{References}

Ambrosi D, Duperray A, Peschetola V, Verdier C. 2009. Traction patterns of tumor cells. J Math Biol. 58:163-181.

Basan M, Prost J, Joanny JF, Elgeti J. 2011. Dissipative particle dynamics simulations for biological tissues: rheology and competition. Phys Biol. 8:026014.

Dieterich P, Klages R, Preuss R, Schwab A. 2008. Anomalous dynamics of cell migration. PNAS. 105(2):459-463.

Drasdo D, Höhme S. 2005. A single-cell-based model of tumor growth in vitro: monolayers and spheroids. Phys Biol. 2:133-147.

Engler A, Sen S, Sweeney H, Discher D. 2006. Matrix elasticity directs stem cell lineage specification. Cell. 126:677-689.

Lauffenburger D, Horwitz A. 1996. Cell migration: a physically integrated molecular process. Cell. 84:359-369.

Lo C, Wang H, Dembo M, Wang Y. 2000. Cell movement is guided by the rigidity of the substrate. Biophys $\mathrm{J}$. 79:144-152.

Peyroux R, Pattana S. 2007. Modelling the mechanical effects accompanying growth and division of cells. Paper presented at: 2nd European Meeting on Cell Mechanics, September 26-2, Barcelona, Spain.

Reffay M, Petitjean L, Coscoy S, Grasland-Mongrain E, Amblard F, Buguin A, Silberzan P. 2011. Orientation and polarity in collectively migrating cell structures: statics and dynamics. Biophys J. 100:2566-2575.

Rørth P. 2011. Whence directionality: guidance mechanisms in solitary and collective cell migration. Dev Cell. 20:9-18.

Sabass B, Gardel M, Waterman C, Schwarz U. 2008. High resolution traction force microscopy based on experimental and computational advances. Biophys J. 94(1):207-220.

Saez A, Ghibaudo M, Buguin A, Silberzan P, Ladoux B. 2007. Rigidity-driven growth and migration of epithelial cells on microstructured anisotropic substrates. PNAS. 104 (20):8281-8286.

Trepat X, Wasserman M, Angelini T, Millet E, Weitz D, Butler J, Fredberg J. 2009. Physical forces during collective cell migration. Nat Phys. 5:426-430.

Trichet L, Le Digabel J, Hawkins R, Vedula S, Gupta M, Ribrault C, Hersen P, Voituriez R, Laboux B. 2012. Evidence of a large scale mechanosensing mechanism for cellular adaptation to substrate stiffness. PNAS. 109(18):6933-6938.

Vermolen F, Gefen A. 2012. A semi-stochastic cell-based formalism to model the dynamics of migration of cells in colonies. Biomech Model Mechanobiol. 11:183-195. 